Article

\title{
Ab Initio-Based Structural and Thermodynamic Aspects of the Electrochemical Lithiation of Silicon Nanoparticles
}

\author{
Seung-Eun Lee ${ }^{1}$, Hyung-Kyu Lim ${ }^{2, *}$ and Sangheon Lee ${ }^{1, *}$ \\ 1 Department of Chemical Engineering and Materials Science, Ewha Womans University, 52, Ewhayeodae-gil, \\ Seodaemun-gu, Seoul 03760, Korea; lsemd96@gmail.com \\ 2 Division of Chemical Engineering and Bioengineering, Kangwon National University, Chuncheon, \\ Gangwon-do 24341, Korea \\ * $\quad$ Correspondence: hklim@kangwon.ac.kr (H.-K.L.); sang@ewha.ac.kr (S.L.); Tel.: +82-33-250-6339 (H.-K.L.); \\ +82-2-3277-4055 (S.L.); Fax: +82-33-259-5551 (H.-K.L.); +82-2-3277-3535 (S.L.)
}

Received: 27 November 2019; Accepted: 16 December 2019; Published: 19 December 2019

check for updates

\begin{abstract}
We reported the theoretical understandings of the detailed structural and thermodynamic mechanism of the actual lithiation process of silicon nanoparticle systems based on atomistic simulation approaches. We found that the rearrangement of the Si bonding network is the key mechanism of the lithiation process, and that it is less frequently broken by lithiation in the smaller sizes of Si nanoparticles. The decreased lithiation ability of the Si nanoparticles results in the lithiation potential being significantly lower than that of crystalline silicon phases, which impedes the full usage of the theoretical maximum capacity. Thus, nanosized Si materials could have a negative effect on performance if they become too fine-sized. These findings provide a detailed view of the electrochemical lithiation process of silicon nanoparticles (Si NPs) and engineering guidelines for designing new Si-based nanostructured materials.
\end{abstract}

Keywords: electrochemical lithiation; silicon nanoparticles; lithiation mechanism; density functional theory; reactive force field

\section{Introduction}

Lithium-ion batteries (LIBs) are among the most predominant energy storage systems for portable to stationary electronic devices. LIBs are indispensable to laptops, mobile phones, and electric vehicles due to their high energy/power density and long cycle life [1]. Accordingly, there is a continuing increase in the technical demand for developing higher capacity/power LIBs. In particular, silicon (Si) has been intensively pursued as one of the most promising anode materials because of its high specific capacity (4200 $\mathrm{mAh} / \mathrm{g}$ for $\mathrm{Li}_{22} \mathrm{Si}_{5}$ ), in comparison with the conventional graphite ( $372 \mathrm{mAh} / \mathrm{g}$ for $\mathrm{LiC}_{6}$ ), and its abundance [2]. Despite its high capacity, Si suffers from fast capacity loss caused by its large volume change ( $>300 \%$ ), unstable solid electrolyte interphase (SEI) and the physical disintegration (cracking and crumbling) of the electrode structure during lithiation/delithiation processes [3,4]. Therefore, there are various research activities to control the electrochemical performance of Si anode materials.

The engineering of Si nanostructures proved to be an effective method for improving capacity and cycling stability, since nano-sized Si can alleviate mechanical fractures during volume changes. It also allows fast charge transfer for a high rate capacity, having a large surface area in contact with the electrolyte [5,6]. There were various types of nanostructured Si materials, including nanoparticles, nano-wires, nano-tubes, and hollow nano-spheres, etc. These nanostructures have been designed and synthesized, and, subsequently, encouraging results have been achieved. Among them, nanoparticles 
have attracted particular interest, since the synthesis of silicon nanoparticles (Si NPs) can feasibly become commercially available, unlike other nano-shaped materials [7]. Moreover, an in-situ transmission electron microscopy (TEM) analysis of Si NPs revealed a strong size dependence of fracture during the first lithiation process, demonstrating that nanoparticles are indeed better in terms of averting the adverse mechanical consequence accompanying electrochemical reactions [8,9]. In this regard, as illustrated in Figure 1, Si NPs can be added into the void region in the polycrystalline graphite matrix, resulting in an effective increase in the overall energy density of the anode.

There has been abundant theoretical research focusing on the detailed lithiation mechanism for Si anode materials using various atomistic scale simulation methods, such as first-principle quantum mechanics (QM) and molecular dynamics (MD) approaches. Furthermore, this research is usually based on the bulk-phase crystal or amorphous silicon materials [10,11]. Several QM studies based on Si NPs have been reported [12,13]. However, due to the high computational burden of the QM method, there is still a lack of systematic address for a theoretical understanding of the lithiation process of various Si NPs. In this work, we designed reasonable structural models of Si NPs in terms of particle size and lithiation ratio through Monte Carlo (MC) simulations, and we conducted a series of first-principle simulations to understand their intrinsic electrochemical properties.

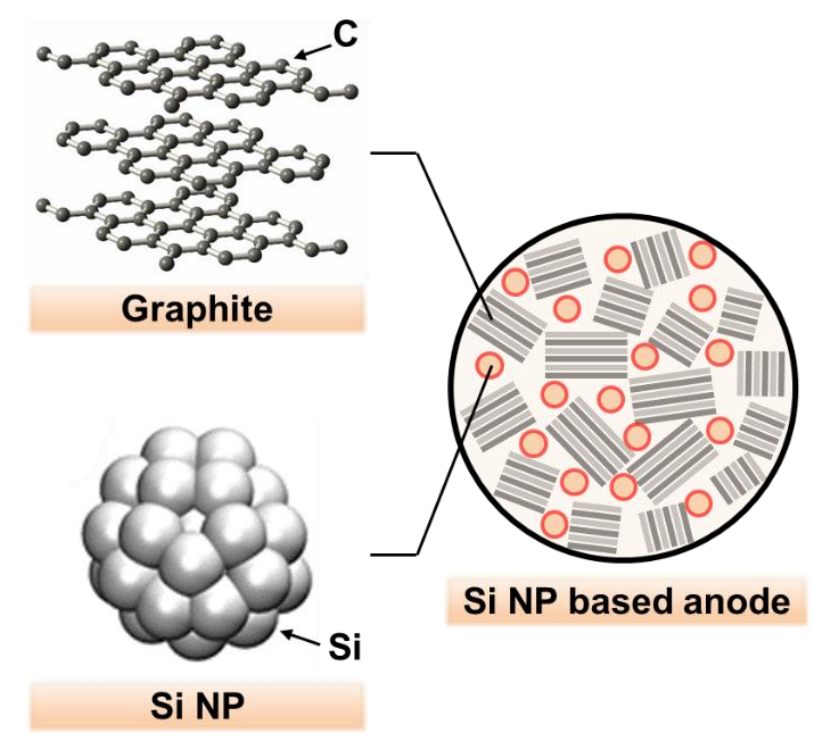

Figure 1. Schematic illustration of a silicon nanoparticle (Si NP) based anode for Li-ion batteries.

\section{Computational Details}

To capture the reasonable atomistic structure of pure and lithiated Silicon nanoparticles using the MC method, we employed the reactive force field (ReaxFF) [14] with the parameters reported by Fan et al., which provided accurate predictions of a set of fundamental properties for $\mathrm{Li}_{x} \mathrm{Si}$ alloys [15]. We considered various atomic structures of pure and lithiated Si NPs in terms of particle size and lithiation ratio, using the in-house MC code, coupled with the Large-scale Atomic/Molecular Massively Parallel Simulator (LAMMPS) [16] software, to evaluate the potential energy based on the ReaxFF parameters. The acceptance of a new trial structure was determined by the Metropolis algorithm. The initial structures of pure and lithiated Si NPs were prepared from the bulk crystalline Si structure, and the lithium atoms at the surface of the optimized final structure of the Si NPs were manually located. To overcome the trapping problem in local minima while searching for a global optimization state, we carried out a simulated annealing process by adjusting the temperature in accordance with the Boltzmann factor of Metropolis criteria from $400 \mathrm{~K}$ to $1600 \mathrm{~K}$ at least five times for each case. The trial move for one temperature annealing cycle was 100,000 times, and the most stable structures of pure and lithiated Si NPs were selected for further analysis. 
The first-principle density functional theory (DFT) calculations were conducted for evaluating statistical atomic distributions and theoretical lithiation potentials of the sampled lithiated Si NP models from the MC step. We used the Vienna Ab Initio Simulation Package (VASP) [17] with Projector Augmented Wave (PAW) type pseudo-potentials for core electrons, and the generalized gradient approximation (GGA) of Perdew-Becke-Ernzerhof (PBE) [18] was used for the exchange-correlation functional. Note that DFT calculations based on the VASP have often been used for the electrochemical characterization of battery electrodes [19-22]. An energy cutoff of $400 \mathrm{eV}$ and gamma-point only k-point sampling schemes were applied for the cluster system of Si NPs. The radial distribution function analysis was conducted using the 5 ps ab initio molecular dynamics (AIMD) trajectory obtained at $300 \mathrm{~K}$ with the Nosé-Hoover thermostat. The radial distribution function (RDF) between $\mathrm{Si}-\mathrm{Si}$ and Si-Li pairs was calculated using the Visual Molecular Dynamics (VMD) [23] software with the following formula:

$$
g(r)=\frac{d n(r)}{\rho \cdot 4 \pi r^{2} d r}
$$

where $d n(r)$ is the number of atoms between $r$ and $r+d r$ from a given atom; $\rho$ is the total number density of the system. We calculated the RDF under a periodic boundary condition with $0.1 \AA$ spacing.

The most stable structures during the AIMD procedure were then fully optimized to evaluate the ground state electronic energy for each lithiated Si NP model. During the geometry optimization, all atoms were fully relaxed using the conjugate gradient method until residual forces on constituent atoms became smaller than $5 \times 10^{-2} \mathrm{eV} / \AA$. From ground state electronic energies for pure and lithiated Si NPs and bulk crystal structures, we derived a theoretical lithiation potential using the following formula:

$$
E_{\text {lithiation }}^{0}\left(\mathrm{~V} \text { vs. } \mathrm{Li}^{+} / \mathrm{Li}\right)=-\left\{E\left(\mathrm{Li}_{x} \mathrm{Si}_{n}\right)-E\left(\mathrm{Li}_{y} \mathrm{Si}_{n}\right)-E\left(\mathrm{Li}_{y-x}\right)\right\} /(y-x)
$$

where $n$ is 18,36 , or $54, E\left(\mathrm{Li}_{x} \mathrm{Si}_{n}\right)$ is the energy of the molecular cluster of pure and lithiated Si NP models, and $E(\mathrm{Li})$ is the energy of the body-centered cubic (BCC) phase of lithium metal.

\section{Results and Discussion}

We produced three optimized pure Si nanoparticle (NP) structures with $18\left(\mathrm{Si}_{18}\right), 36\left(\mathrm{Si}_{36}\right)$, and 54 ( $\left.\mathrm{Si}_{54}\right) \mathrm{Si}$ atoms using the MC sampling method based on the ReaxFF potential (Figure 2a). The sizes of the Si NPs were approximately $0.7,1.0$, and $1.3 \mathrm{~nm}$ for $\mathrm{Si}_{18}, \mathrm{Si}_{36}$, and $\mathrm{Si}_{54}$, respectively, including the Shannon radii for Si atoms $(0.04 \mathrm{~nm})$ [24]. We found that the structures of Si NPs are highly symmetrized and that these Si-Si bonding networks are closer to the bulk amorphous Si structure (a-Si) than to the bulk crystalline Si structure (c-Si). As shown in Figure 2b,c, the bond and angle distributions of the nearest neighbor Si atoms clearly show the structural similarity between Si NPs and a-Si (the structure of a-Si in Figure 2a was obtained from other literature [25]). As the size of the Si NP increases from $\mathrm{Si}_{18}$ to $\mathrm{Si}_{54}$, the overall distances of $\mathrm{Si}-\mathrm{Si}$ bonds are shortened, getting close to the distribution of the a-Si (the average $\mathrm{Si}-\mathrm{Si}$ bond distances are 2.50, 2.48, 2.47, 2.38, and $2.35 \AA$ for $\mathrm{Si}_{18}, \mathrm{Si}_{36}, \mathrm{Si}_{54}, \mathrm{a}-\mathrm{Si}$, and c-Si, respectively), by the decreased contribution of the surface $\mathrm{Si}$ atoms that have low coordination numbers (the Connolly surface [26] to atomic volume ratios are $0.69,0.58$, and $0.52 \AA^{-1}$ for $\mathrm{Si}_{18}, \mathrm{Si}_{36}$, and $\mathrm{Si}_{54}$, respectively). For the same reason, the angle distributions around $60^{\circ}$ (contributions from the surface $\mathrm{Si}$ atoms) are decreased as the size of the NPs increases. The rest of the angle distribution shows an almost similar shape to that of a-Si (average angles are 90.83, 95.99, $97.59,109.02$, and $109.47^{\circ}$ for $\mathrm{Si}_{18}, \mathrm{Si}_{36}, \mathrm{Si}_{54}$, a-Si, and c-Si, respectively). Due to the nature of this $\mathrm{Si}$ bonding network, the lithiation behavior of Si NPs could be closer to a-Si than c-Si so that the faster Li diffusion is expected inside the Si NPs, as reported in previous theoretical studies [27-29] that are based on bulk a-Si structures. In this regard, we theoretically investigated the lithiation behavior in $\mathrm{Si}$ NP models with high surface ratios. 
$\mathbf{a}$
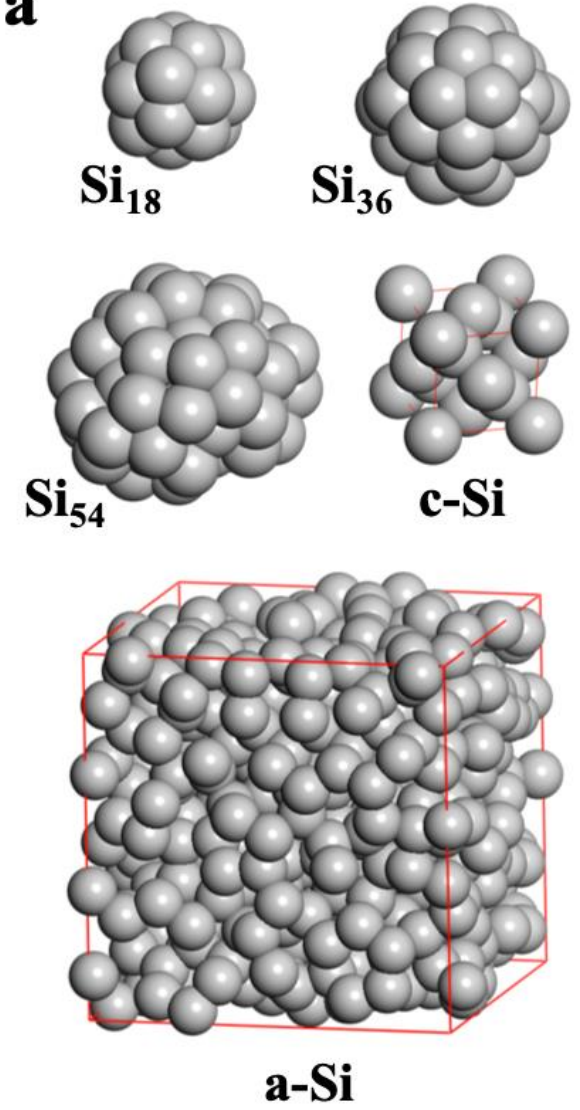

b
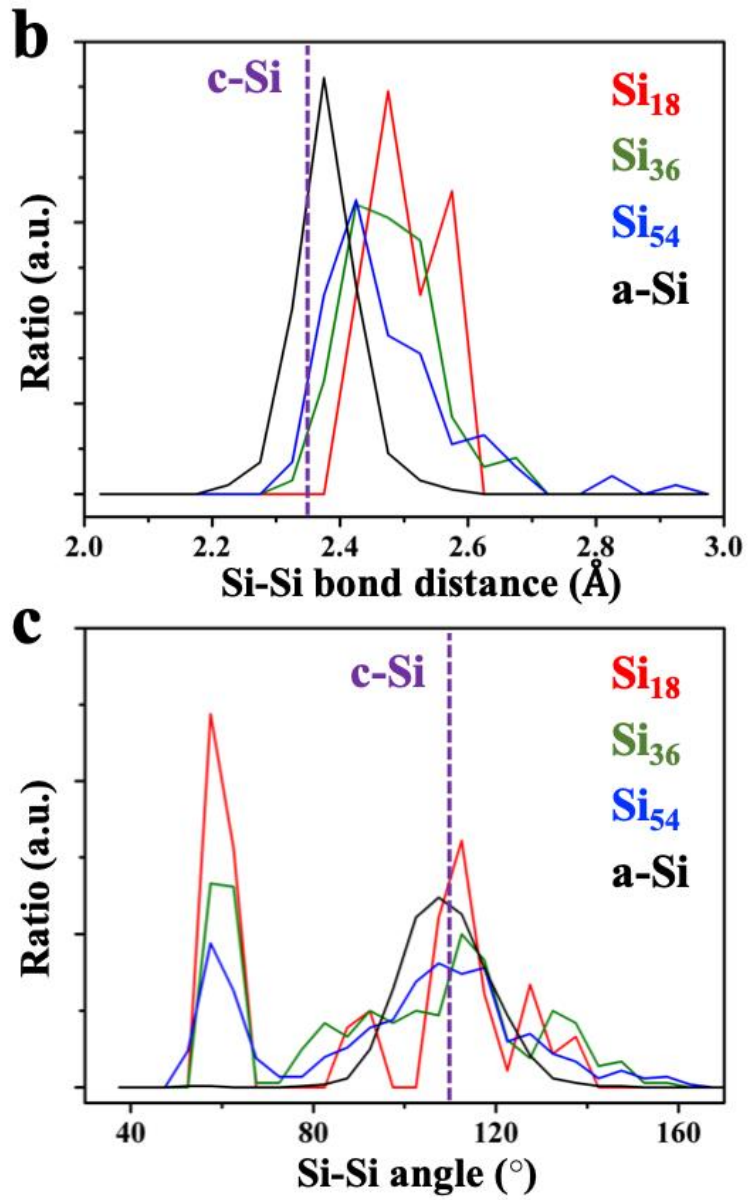

Figure 2. (a) The optimized structures of Si NPs using the Monte Carlo (MC) sampling method based on the reactive force field (ReaxFF) potential, and the unit cell structures of crystalline and amorphous bulk silicon. $(\mathbf{b}, \mathbf{c})$ The distributions of bond distances and angles between the nearest neighbor $\mathrm{Si}$ atoms inside the Si NPs and bulk phases.

Based on the three Si NP structures, we sampled stable lithiated structures in terms of a lithium to silicon (L-S) ratio, using the MC sampling method based on the ReaxFF potential. We produced lithiated structures of Si NPs with an L-S ratio of 1.0 to 4.0. In particular, for the smallest $\mathrm{Si}_{18}$ structure, we finely generated the lithiated Si NPs with an L-S ratio of 0.1 to 0.8 to clearly observe the characteristics of the initial lithiation process of the $\mathrm{Si}$ NP. The most stable lithiated $\mathrm{Si}_{18}$ structures from an $L-S$ ratio of 0.1 to 4.0 are shown in Figure 3. At the initial stage of the lithiation process $(0.1 \sim 0.3$ of $\mathrm{L}-\mathrm{S}$ ratio) of $\mathrm{Si}_{18}$, $\mathrm{Si}$ NPs retain the main bonding network, almost similar to that of the original one, and most of the lithium atoms tend to form a cluster at the outer surface. At the L-S ratio of 0.2 and 0.3 , Si NP structures with one lithium atom penetrated into the center were sampled to be stable. In the real lithiation process, however, the penetration of a single lithium atom into the center site would require a considerable equilibrium temperature and time because the lithium diffusion through a compact triangular or rectangular Si bonding network has a large thermodynamic barrier. 


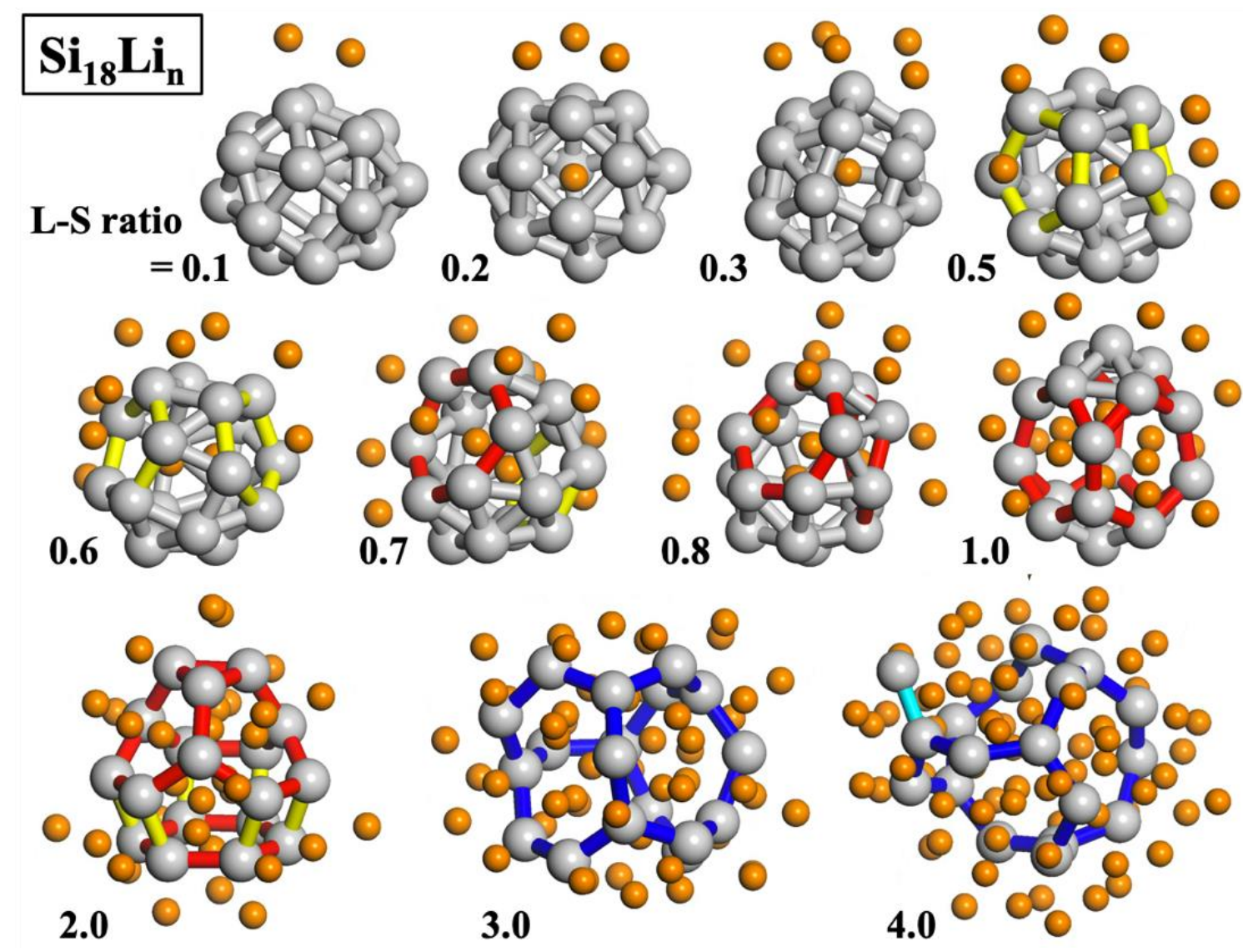

Figure 3. The most stable lithiated $\mathrm{Si}_{18}$ structures in terms of a lithium to silicon ratio of 0.1 to 4.0. $\mathrm{Si}$ atoms are colored in gray and $\mathrm{Li}$ atoms are colored in orange. The type of ring consisting of $\mathrm{Si}$ bonds is colored with yellow for pentagon, red for hexagon, and blue for more than heptagon. The cyan color indicates the dangling Si bond.

For the L-S ratio of 0.5 and above, lithium insertion results in significant change in the electronic structure of a Si NP, accompanied by change in the Si bonding network. For instance, the original Si bonding networks of the triangles and squares of $\mathrm{Si}_{18}$ expand to form pentagonal, hexagonal, and more than heptagonal (yellow, red, and blue colored bonds in Figure 3, respectively) Si rings as the number of lithium atoms penetrated into the particle center increases. Facile lithium diffusion could occur through the systematically expanded Si bonding networks, with two expanded Si rings (pentagons or hexagons) in an L-S ratio between 0.5 and 0.8 , three hexagons in 1.0, and four hexagons and three pentagons in 2.0. In the L-S ratio of 3.0 and 4.0, heptagonal or greater Si rings are formed due to the greatly weakened $\mathrm{Si}-\mathrm{Si}$ bonds by lithiation and start to generate a dangling $\mathrm{Si}$ bond (cyan colored bond in Figure 3) in the final stage of lithiation.

For the $\mathrm{Si}$ NPs larger than $\mathrm{Si}_{18}$, bonds between $\mathrm{Si}$ atoms are more easily broken during lithiation. The most stable sampled structures for $\mathrm{Si}_{36}$ and $\mathrm{Si}_{54}$ during the lithiation are shown in Figure 4. For the $\mathrm{Si}_{36}$ case, dangling $\mathrm{Si}$ bonds start to form at an $\mathrm{L}-\mathrm{S}$ ratio of 3.0, and subsequent $\mathrm{Si}-\mathrm{Si}$ bond breaking occurs at 4.0, thereby resulting in a large number of $\mathrm{Si}$ dumbbells and isolated $\mathrm{Si}$ atoms surrounded by $\mathrm{Li}$ atoms. In the case of $\mathrm{Si}_{54}$, $\mathrm{Si}$ dangling bonds form from a lower $\mathrm{L}-\mathrm{S}$ ratio of 2.0, and a large number of $\mathrm{Si}$ dumbbells and isolated $\mathrm{Si}$ atoms form at 4.0. As shown in Figure 5, this tendency to break the $\mathrm{Si}$ bonding network is even more prominent in the fully equilibrated bulk-phase lithiated silicon crystal structures, where the number of $\mathrm{Si}$ atoms constituting the $\mathrm{Si}$ clusters in the crystal gradually decreases as the L-S ratio increases from 1.7 to 3.8. Therefore, the Si bonding network in a Si NP structure is relatively hard to break compared to the bulk structure, because the unstable Si atoms formed after the breakage of the Si network cannot be sufficiently stabilized by the lithium atoms on the particle 
surface. From these structural characteristics of the lithiation process of extremely fine Si NPs, it would seem that a certain level of content in an anode could be one of the reasons for failing to achieve the theoretical capacity. Furthermore, it could drive undesired metal plating on the surface of Si NPs, possibly causing lithium dendrite formation [30].

a

L-S ratio $=\mathbf{1 . 0}$
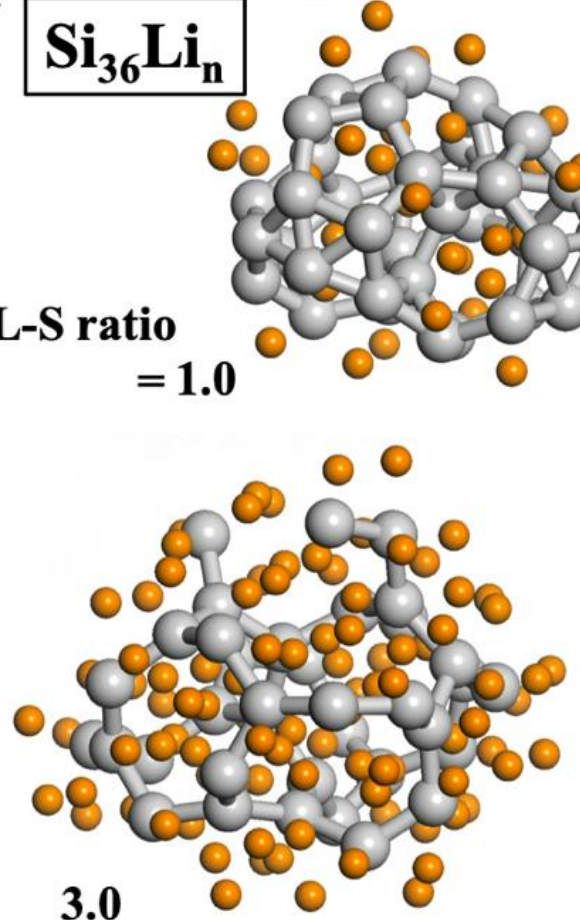

$\mathbf{b} \mathbf{S i}_{54} \mathbf{L} \mathbf{i}_{\mathbf{n}}$
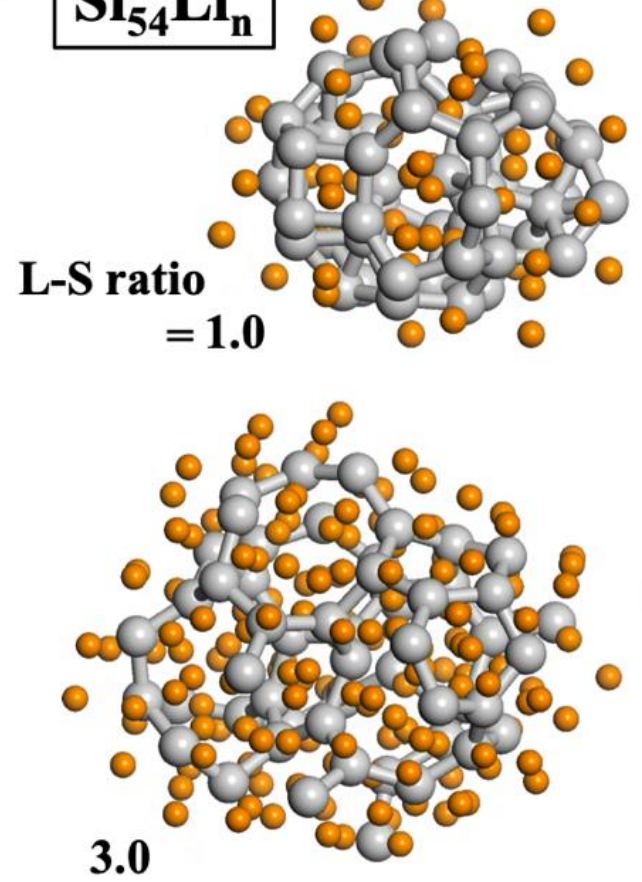
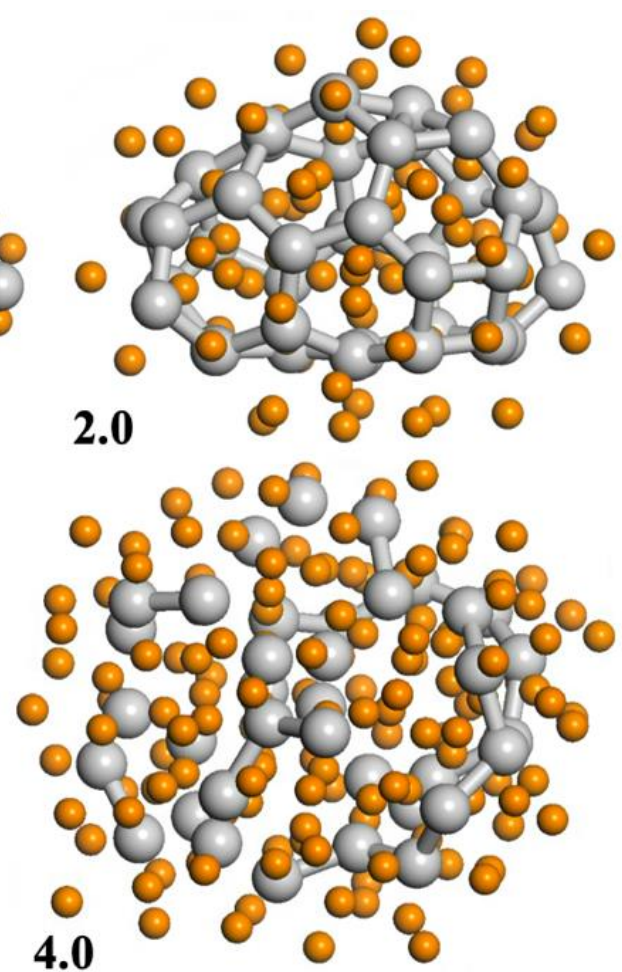

4.0

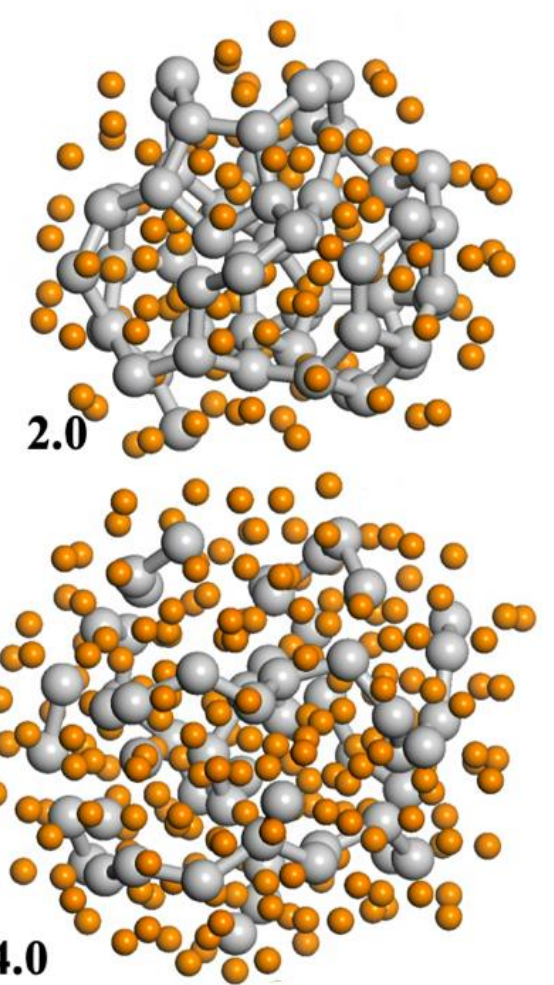

Figure 4. The most stable lithiated (a) $\mathrm{Si}_{36}$ and (b) $\mathrm{Si}_{54}$ structures in terms of a lithium to silicon ratio of 1.0 to 4.0. Si atoms are colored in gray and $\mathrm{Li}$ atoms are colored in orange. 


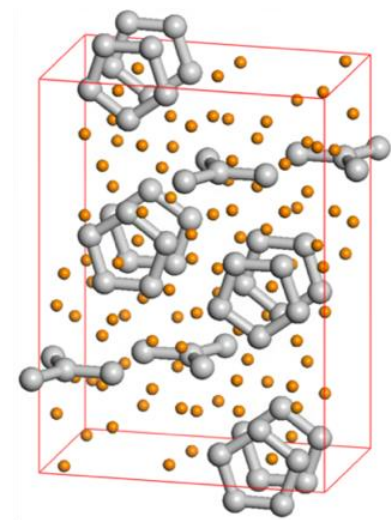

$\mathrm{Si}_{7} \mathbf{L i}_{12}(\mathbf{1 . 7})$

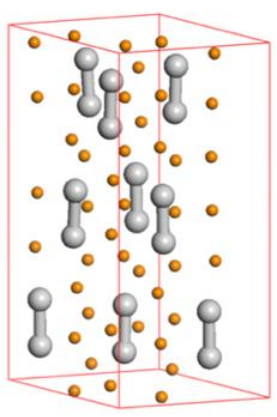

$\mathrm{Si}_{3} \mathbf{L i}_{7}(2.3)$

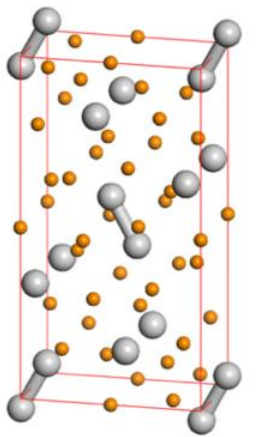

$\mathrm{Si}_{4} \mathbf{L i}_{13}(3.3)$

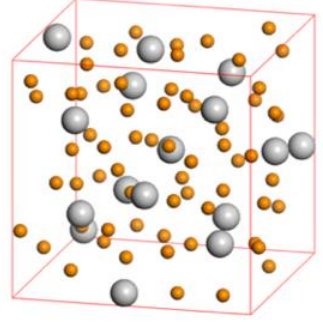

$\mathrm{Si}_{4} \mathbf{L i}_{15}(\mathbf{3 . 8 )}$

Figure 5. The crystal structures for bulk-phase lithiated Si materials, showing the broken Si bonding network in terms of the degree of lithiation. The numbers in parentheses refer to the lithium to silicon $(\mathrm{L}-\mathrm{S})$ ratio.

For each sampled lithiated Si NP from the MC simulations, we also performed DFT calculations to analyze more precise structural and thermodynamic characteristics. First, we analyzed the radial distribution function (RDF) between atomic pairs inside $\mathrm{Si}_{18}, \mathrm{Si}_{36}$, and $\mathrm{Si}_{54} \mathrm{NPs}$, using the L-S ratio 1.0 to 4.0, by obtaining ab initio molecular dynamics (AIMD) trajectories at $300 \mathrm{~K}$ (Figure 6). From the position of the first peak of RDF, the bond distances and the coordination numbers between the nearest neighbor atoms of $\mathrm{Si}-\mathrm{Si}$ and $\mathrm{Si}-\mathrm{Li}$ pairs are almost unchanged during the lithiation of all $\mathrm{Si}$ NPs. The second nearest neighbor distribution patterns of Si-Si RDF show a tendency to gradually increase the $\mathrm{Si}-\mathrm{Si}$ distance and decrease the broadness of the $\mathrm{Si}-\mathrm{Si}$ distance distribution depending on the degree of lithiation. Considering that this tendency is obvious in large size Si NPs, the extent to which the Si bonding network is broken to form isolated silicon dumbbells or atoms occurs dominantly in the large Si NPs, as observed in the above MC simulation results.
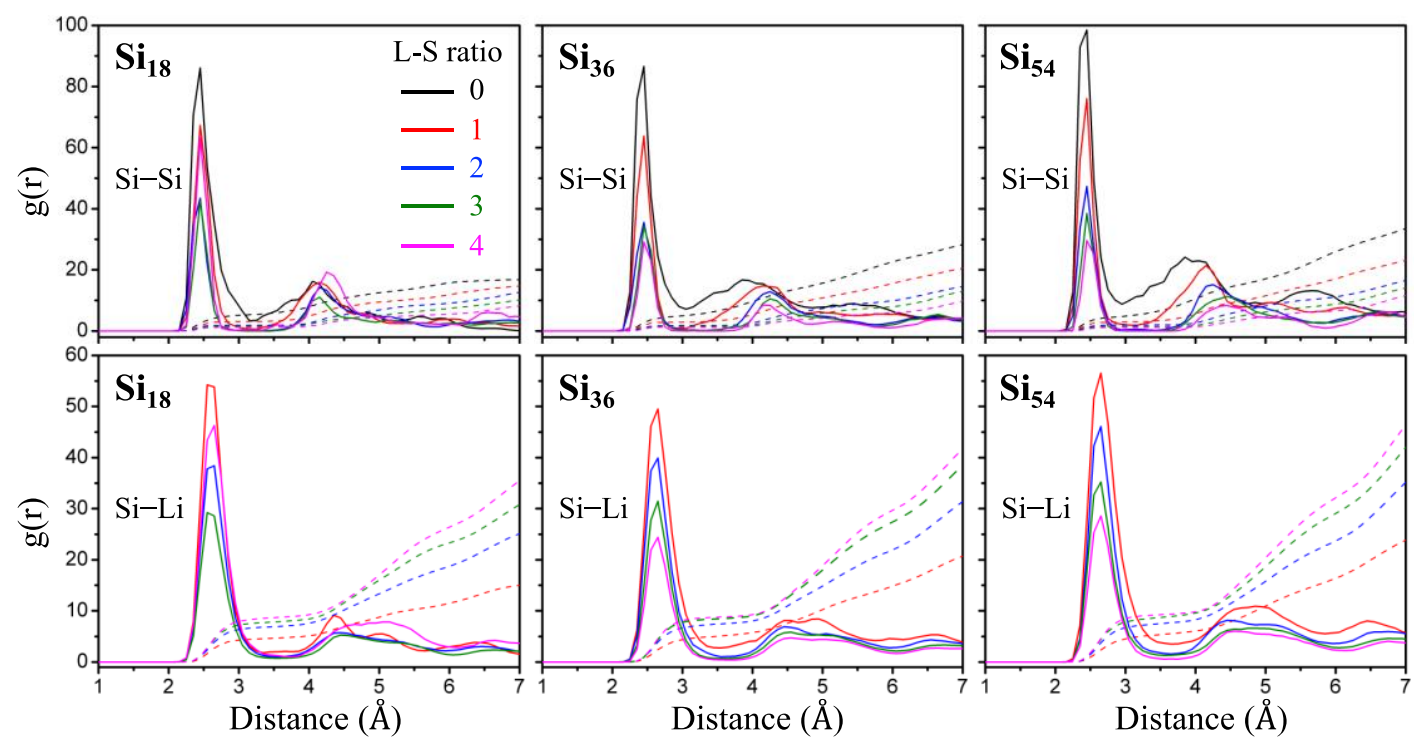

Figure 6. The radial distribution functions for $\mathrm{Si}-\mathrm{Si}$ and $\mathrm{Si}-\mathrm{Li}$ atomic pairs for each lithiated Si NP during the $300 \mathrm{~K} \mathrm{ab}$ initio molecular dynamics (AIMD) simulations. The solid lines are the radial distribution function (RDF), and the dashed lines are the integral values.

Next, we estimated the theoretical lithiation potential profile of Si NPs by calculating the ground-state DFT energies based on the most stable structures captured during the AIMD simulations and compared them with the corresponding values from the homogeneous bulk lithiated silicon crystal 
structures $\left(\mathrm{Si}_{7} \mathrm{Li}_{12}, \mathrm{Si}_{3} \mathrm{Li}_{7}, \mathrm{Si}_{4} \mathrm{Li}_{13}\right.$, and $\mathrm{Si}_{4} \mathrm{Li}_{15}$ phases in Figure 5 and the additional $\mathrm{Si}_{5} \mathrm{Li}_{21}$ phase). As shown in Figure 7, the lithiation (charging process) potential profiles of Si NPs are considerably lower than those of ideal bulk crystals because of the overall nanosized effect including the insufficient breakage of Si bonding networks. Specifically, it is expected that the lithiation capacity of Si NPs will not be able to obtain more than $3 / 4$ of the bulk-phase capacity because the lithiation potentials become less than $0 \mathrm{~V}$ before the $\mathrm{L}-\mathrm{S}$ ratio of 3 . As discussed earlier, if the lithiation potential is lowered below $0 \mathrm{~V}$ versus the Li reduction potential, lithium metal deposition on the surface of Si NPs could be feasible and therefore the lithium dendrite could be easily formed on the surface of Si NPs, which may adversely affect LIB operations. This tendency is expected to be greater as the size of the Si NP is further reduced. There is little difference between $\mathrm{Si}_{54}$ and $\mathrm{Si}_{36} \mathrm{NPs}$, but for the all $\mathrm{L}-\mathrm{S}$ ratios, their lithiation potentials increase by about $0.1 \mathrm{~V}$ in comparison with the $\mathrm{Si}_{18}$ case. Compared to the larger $\mathrm{Si} N$ Ps, this clearly reflects a characteristic of the hard-to-break $\mathrm{Si}$ bonding network of $\mathrm{Si}_{18}$ observed through lithiated Si NP structures from MC simulations. Since the actual lithiation potential of a Si $\mathrm{NP}$ is expected to be lower due to the various overpotentials in actual battery operation, the same result can be expected in Si NPs larger than the size covered in this study. Therefore, more in-depth experimental and theoretical studies on the side effects of the nanonization of silicon materials on the performance of LIBs are necessary.

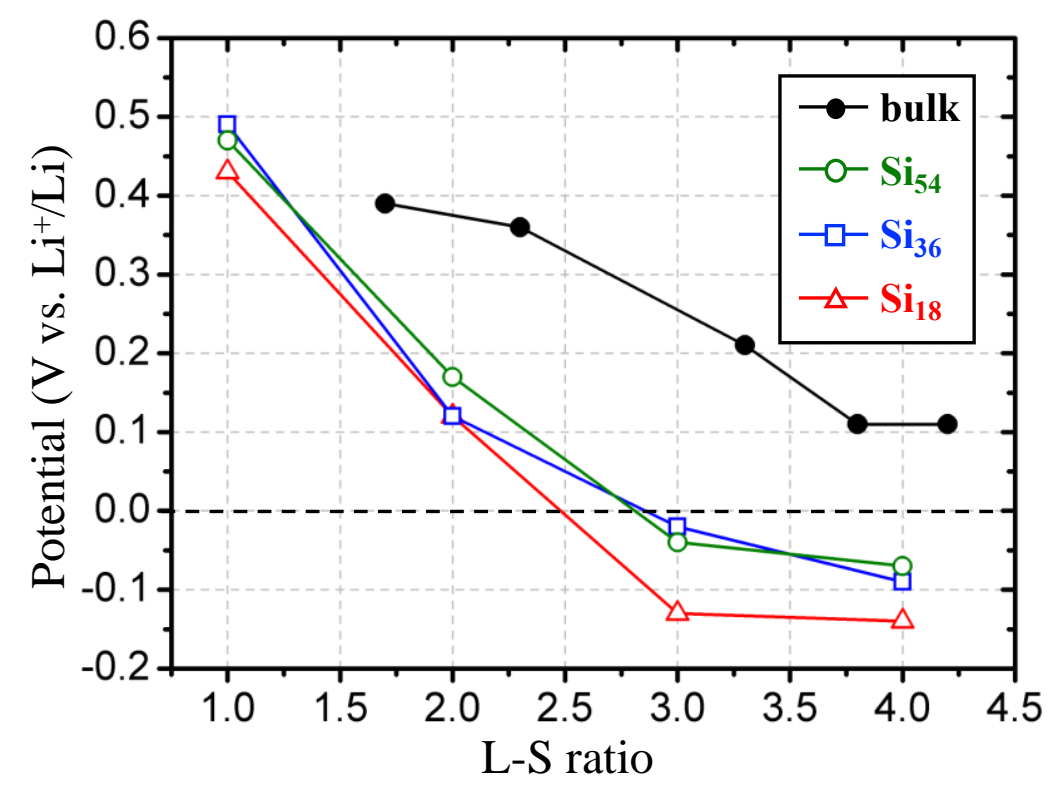

Figure 7. The theoretical lithiation potential for Si NPs and bulk crystalline Si materials calculated from the first-principle density functional theory (DFT) simulations.

\section{Conclusions}

In summary, the structural and energetic properties of the actual lithiation process were investigated using the extremely fine Si NP models based on the ReaxFF and first-principle DFT simulation methods. The larger the Si NP size (the lower the surface-to-volume ratio), the easier the Si bonding network is broken by lithiation to form a more homogeneous lithiated silicon structure. This decreased lithiation ability of the Si bonding network of Si NPs results in the theoretical lithiation potential being significantly lower than that of ideal bulk-phase lithiated silicon crystals, which falls below the reduction potential of lithium metal before the L-S ratio of 3 . Therefore, when such extremely fine $\mathrm{Si}$ NPs are present in the electrode, it becomes impossible to achieve the theoretically possible maximum capacity of $\mathrm{Si}$ anodes. In addition, the facile formation of the lithium metal phases on the surface of $\mathrm{Si}$ NPs can lead to the formation of lithium dendrites, which may adversely affect the stable operation of the Li-ion battery. From the theoretical understanding of the lithiation mechanism of Si NPs, it can be seen that the control of particle size could be a very important factor in the LIB manufacturing 
process. Future research will be able to suggest a nanoparticle size for optimal performance by studying lithiation behavior over a wider range of nanoparticle sizes.

Author Contributions: Conceptualization, H.-K.L.; Data curation, S.-E.L.; Formal analysis, S.-E.L.; Funding acquisition, H.-K.L. and S.L.; Supervision, H.-K.L. and S.L.; Writing-original draft, S.-E.L.; Writing-review \& editing, H.-K.L. and S.L. All authors have read and agreed to the published version of the manuscript.

Funding: This research was funded by the National Research Foundation of Korea NRF-2018R1D1A1B07051430, and by the Technology Development Program to Solve Climate Changes through the National Research Foundation of Korea NRF-2018M1A2A2061994. This work was also supported by the 2019 Research Grant from Kangwon National University.

Conflicts of Interest: The authors declare no conflict of interest.

\section{References}

1. Goodenough, J.B.; Park, K.-S. The Li-Ion Rechargeable Battery: A Perspective. J. Am. Chem. Soc. 2013, 135, 1167-1176. [CrossRef]

2. Winter, M.; Besenhard, J.O. Electrochemical Lithiation of Tin and Tin-Based Intermetallics and Composites. Electrochim. Acta 1999, 45, 31-50. [CrossRef]

3. Ma, D.; Cao, Z.; Hu, A. Si-Based Anode Materials for Li-Ion Batteries: A Mini Review. Nano-Micro Lett. 2014, 6, 347-358. [CrossRef] [PubMed]

4. Zuo, X.; Zhu, J.; Müller-Buschbaum, P.; Cheng, Y.-J. Silicon Based Lithium-Ion Battery Anodes: A Chronicle Perspective Review. Nano Energy 2017, 31, 113-143. [CrossRef]

5. Goriparti, S.; Miele, E.; De Angelis, F.; Di Fabrizio, E.; Proietti Zaccaria, R.; Capiglia, C. Review on Recent Progress of Nanostructured Anode Materials for Li-Ion Batteries. J. Power Sources 2014, 257, 421-443. [CrossRef]

6. Xu, Z.-L.; Liu, X.; Luo, Y.; Zhou, L.; Kim, J.-K. Nanosilicon Anodes for High Performance Rechargeable Batteries. Prog. Mater. Sci. 2017, 90, 1-44. [CrossRef]

7. Yin, S.; Ji, Q.; Zuo, X.; Xie, S.; Fang, K.; Xia, Y.; Li, J.; Qiu, B.; Wang, M.; Ban, J.; et al. Silicon Lithium-Ion Battery Anode with Enhanced Performance: Multiple Effects of Silver Nanoparticles. J. Mater. Sci. Technol. 2018, 34, 1902-1911. [CrossRef]

8. Liu, X.H.; Liu, Y.; Kushima, A.; Zhang, S.; Zhu, T.; Li, J.; Huang, J.Y. In Situ TEM Experiments of Electrochemical Lithiation and Delithiation of Individual Nanostructures. Adv. Energy Mater. 2012, 2, 722-741. [CrossRef]

9. $\mathrm{Su}, \mathrm{X} . ; \mathrm{Wu}, \mathrm{Q}$; Li, J.; Xiao, X.; Lott, A.; Lu, W.; Sheldon, B.W.; Wu, J. Silicon-Based Nanomaterials for Lithium-Ion Batteries: A Review. Adv. Energy Mater. 2014, 4, 1300882. [CrossRef]

10. Chan, M.K.Y.; Wolverton, C.; Greeley, J.P. First Principles Simulations of the Electrochemical Lithiation and Delithiation of Faceted Crystalline Silicon. J. Am. Chem. Soc. 2012, 134, 14362-14374. [CrossRef]

11. Van Duin, A.C.T.; Strachan, A.; Stewman, S.; Zhang, Q.; Xu, X.; Goddard, W.A. ReaxFF SiO Reactive Force Field for Silicon and Silicon Oxide Systems. J. Phys. Chem. A 2003, 107, 3803-3811. [CrossRef]

12. Hankins, K.; Soto, F.A.; Balbuena, P.B. Insights into the Li Intercalation and SEI Formation on LiSi Nanoclusters. J. Electrochem. Soc. 2017, 164, E3457-E3464. [CrossRef]

13. Okamoto, Y. Dynamical Aspects of Lithiation of a Nanosized Silicon Cluster. J. Phys. Chem. C 2011, 115, 25160-25164. [CrossRef]

14. Van Duin, A.C.T.; Dasgupta, S.; Lorant, F.; Goddard, W.A. ReaxFF: A Reactive Force Field for Hydrocarbons. J. Phys. Chem. A 2001, 105, 9396-9409. [CrossRef]

15. Fan, F.; Huang, S.; Yang, H.; Raju, M.; Datta, D.; Shenoy, V.B.; van Duin, A.C.T.; Zhang, S.; Zhu, T. Mechanical Properties of Amorphous $\mathrm{Li}_{x} \mathrm{Si}$ Alloys: A Reactive Force Field Study. Model. Simul. Mater. Sci. Eng. 2013, 21, 074002. [CrossRef]

16. Plimpton, S. Fast Parallel Algorithms for Short-Range Molecular Dynamics. J. Comput. Phys. 1995, 117, 1-19. [CrossRef]

17. Kresse, G.; Hafner, J. Ab Initio Molecular Dynamics for Liquid Metals. Phys. Rev. B 1993, 47, 558-561. [CrossRef]

18. Perdew, J.P.; Burke, K.; Ernzerhof, M. Generalized Gradient Approximation Made Simple. Phys. Rev. Lett. 1996, 77, 3865-3868. [CrossRef] 
19. Minakshi, M.; Mitchell, D.R.G.; Baur, C.; Chable, J.; Barlow, A.J.; Fichtner, M.; Banerjee, A.; Chakraborty, S.; Ahuja, R. Phase Evolution in Calcium Molybdate Nanoparticles as a Function of Synthesis Temperature and Its Electrochemical Effect on Energy Storage. Nanoscale Adv. 2019, 1, 565-580. [CrossRef]

20. Watcharatharapong, T.; Minakshi Sundaram, M.; Chakraborty, S.; Li, D.; Shafiullah, G.; Aughterson, R.D.; Ahuja, R. Effect of Transition Metal Cations on Stability Enhancement for Molybdate-Based Hybrid Supercapacitor. ACS Appl. Mater. Interfaces 2017, 9, 17977-17991. [CrossRef]

21. Chou, C.-Y.; Lee, M.; Hwang, G.S. A Comparative First-Principles Study on Sodiation of Silicon, Germanium, and Tin for Sodium-Ion Batteries. J. Phys. Chem. C 2015, 119, 14843-14850. [CrossRef]

22. Chou, C.-Y.; Kim, H.; Hwang, G.S. A Comparative First-Principles Study of the Structure, Energetics, and Properties of Li-M (M = Si, Ge, Sn) Alloys. J. Phys. Chem. C 2011, 115, 20018-20026. [CrossRef]

23. Humphrey, W.; Dalke, A.; Schulten, K. VMD: Visual Molecular Dynamics. J. Mol. Graph. 1996, 14, $33-38$. [CrossRef]

24. Shannon, R.D. Revised Effective Ionic Radii and Systematic Studies of Interatomic Distances in Halides and Chalcogenides. Acta Crystallogr. Sect. A 1976, 32, 751-767. [CrossRef]

25. Deringer, V.L.; Bernstein, N.; Bartók, A.P.; Cliffe, M.J.; Kerber, R.N.; Marbella, L.E.; Grey, C.P.; Elliott, S.R.; Csányi, G. Realistic Atomistic Structure of Amorphous Silicon from Machine-Learning-Driven Molecular Dynamics. J. Phys. Chem. Lett. 2018, 9, 2879-2885. [CrossRef]

26. Connolly, M.L. Analytical Molecular Surface Calculation. J. Appl. Crystallogr. 1983, 16, 548-558. [CrossRef]

27. Johari, P.; Qi, Y.; Shenoy, V.B. The Mixing Mechanism during Lithiation of Si Negative Electrode in Li-Ion Batteries: An Ab Initio Molecular Dynamics Study. Nano Lett. 2011, 11, 5494-5500. [CrossRef]

28. Tritsaris, G.A.; Zhao, K.; Okeke, O.U.; Kaxiras, E. Diffusion of Lithium in Bulk Amorphous Silicon: A Theoretical Study. J. Phys. Chem. C 2012, 116, 22212-22216. [CrossRef]

29. Huang, S.; Zhu, T. Atomistic Mechanisms of Lithium Insertion in Amorphous Silicon. J. Power Sources 2011, 196, 3664-3668. [CrossRef]

30. Li, X.; Gu, M.; Hu, S.; Kennard, R.; Yan, P.; Chen, X.; Wang, C.; Sailor, M.J.; Zhang, J.-G.; Liu, J. Mesoporous Silicon Sponge as an Anti-Pulverization Structure for High-Performance Lithium-Ion Battery Anodes. Nat. Commun. 2014, 5, 4105. [CrossRef] 